Ann. Zootech., I979, 28 (I), 53-72.

\title{
Qualités organoleptiques des viandes de Porcs mâles entiers ou castrés : composition des graisses et odeurs sexuelles chez les races hypermusclées
}

\author{
M. BONNEAU, B. DESMOULIN, B. L. DUMONT (*) \\ Avec la collaboration technique de MM. Chalier, Peiniac, Gransart, Dechaset et Lexis (I) \\ Mmes Cano, Degas, Hudzik, Paumier et Roussel. $\left(^{*}\right)$ \\ Station de Recherches sur l'Elevage des Porcs, \\ (*) Laboratoire de Recherches sur la Viande, \\ Centre national de Recherches Zootechniques, I.N.R.A. \\ 78350 Jouy-en-Josas (France)
}

\begin{abstract}
Résumé
Nous arons étudié la composition des graisses en relation avec les défauts d'odeur sexuelle et les qualités organoleptiques des viandes de porcs mâles entiers ou castrés de race Landrace Belge ou Piétrain.

La teneur en androsténone ( $5 \alpha$ androst-I6-ene-3-one) des graisses dorsales des porcs mâles entiers présente une très forte variabilité intrarace. Chez le Piétrain, la teneur moyenne en stéroïde ( $1,75 \mathrm{ppm})$ est supérieure à celle des Landrace Belge $(0,6 \mathrm{r} \mathrm{ppm})$. Par ailleurs, la teneur en acide linoléique est plus élevée chez les mâles entiers par rapport aux castrats et chez les Piétrain par rapport aux Landrace Belge, en liaison négative étroite $(r=-0,86 \grave{a}-0,89)$ avec le degré d'adiposité des carcasses.

La notation de l'intensité des odeurs sexuelles est réalisée par un jury de laboratoire; elle est reliée positivement avec la teneur en androsténone des graisses lors du chauffage rapide du tissu gras dorsal au fer à souder $\left(r_{\varepsilon}=+0,4 \mathrm{I} \grave{\mathrm{a}}+0,57\right)$ et lors de la cuisson des rôtis $\left(r_{8}=+\right.$ $0,44$ à 0,54$)$. Les limites de l'appréciation olfactive des graisses et des viandes sont liées d'une part à la nature des tests pratiqués et d'autre part à la contribution probable de composés autres que l'androsténone. La fréquence des odeurs sexuelles est très importante dès $90 \mathrm{~kg}$ de poids vif chez les Piétrain. Elle est faible à ce même stade chez les Landrace Belge mais augmente nettement à I I o kg.

Lors de la consommation en frais, les viandes de porcs mâles entiers sont jugées plus défavorablement que celles des castrats aussi bien par le jury de laboratoire ( 30 à 60 p. roo d'avis défavorables) que par le jury de consommateurs ( 5 à $30 \mathrm{p}$. roo des avis).

Après transformation en jambon cuit, les différences entre types sexuels ne sont perçues par aucun des deux jury. Par contre, dans le cas des rillettes et du saucisson cuit, les viandes de porcs mâles entiers sont jugées plus défavorabiement que celles des castrats par le jury de laboratoire.
\end{abstract}




\section{Introduction}

Les graisses sont le principal support des composés responsables de l'odeur de cuisson des viandes et de la flaveur au cours de la dégustation. Dans le cas des viandes de porcs mâles entiers, le stockage de stéroïdes sexuels dans les graisses est à l'origine d'odeurs désagréables dites "odeurs sexuelles " apparentées à celles d'oignon ou de sueur (LERCHE, I 936) ou encore d'urine (HoRst et BADER, I969).

Le principal composé responsable des odeurs sexuelles est l'androsténone (5 $x$ androst-I6-ene-3-one) (PATTERSON, I968). Les quantités de ce stéroïde stockées dans le tissu gras peuvent varier considérablement de 0,2 à $5 \mathrm{ppm}$. ANDRESEN et BAKKE (I 975) ont même décelé 42 , I ppm dans le tissu gras d'un verrat. La fréquence des odeurs sexuelles prononcées est relativement faible (moins de 5 p. Ioo) chez les animaux de type Large White élevés en loges individuelles (DEsmoutin, Dumont et Jacgues, i97I; Desmoulin, Dumont et Pascal, i973; Dumont et Desmoulın, I972). Peu d'études ont été effectuées sur les mâles entiers des races de porcs hypermusclés de type Landrace Belge et Piétrain largement utilisés dans les schémas de croisement. CASTEELs et al. (r974) ont montré que, chez ces animaux, I 5 à $20 \mathrm{p}$. Ioo des odeurs des graisses chauffées sont anormalement prononcées.

Dans le cadre d'un essai consacré à l'analyse de l'influence de la castration sur les performances zootechniques et la composition corporelle dans les races hypermusclées (DESMOUlin et BonNeAU, I979), on a considéré la fréquence et l'intensité des défauts d'odeurs des viandes. Les relations entre la teneur en androsténone des graisses et leurs propriétés sensorielles ont été explorées. En outre, nous avons étudié les propriétés sensorielles des viandes fraîches et des produits transformés qui en dérivent.

\section{Matériel et méthodes}

\section{I. - Animaux et dispositif expérimental}

Soixante douze porcs mâles de race Piétrain (PP) ou Landrace Belge (LB) sont répartis en couples d'animaux de même portée. L'un des mâles est castré, l'autre restant entier. La castration ayant été effectuée vers I 8 à $20 \mathrm{~kg}$ de poids vif dans les élevages d'origine, les porcs sont alimentés à volonté en loge individuelle selon les conditions définies par ailleurs (DESMOULIN et BONNEAU, I979). Ils sont affectés à des stades d'abattages léger ( $90 \mathrm{~kg}$ ) ou lourd (IOO-I Io kg). Le dispositif expérimental factoriel $2 \times 2 \times 2$ comporte l'étude des effets de la race, de la castration et du poids d'abattage.

\section{II. - Analyse de la composition des graisses}

La composition en acides gras des graisses dorsales et périrénales a été déterminée pour 30 animaux ( 6 mâles entiers et $\mathrm{I} 4$ castrats) selon une technique décrite par Pascal et Desmoulin (I973).

Par ailleurs, la teneur en androsténone du tissu gras dorsal a été mesurée chez les porcs mâles entiers selon la méthode mise au point par CraUs (I974). 
$40 \mathrm{mg}$ de graisses sont extraits sous reflux par de l'éther de pétrole. La présence de lipides perturbant la suite du dosage, l'androsténone est ensuite extraite par du méthanol. Une partie aliquote de l'extrait méthanolique est alors utilisée pour le dosage radioimmunologique de l'androsténone. L'antisérum spécifique dirigé contre 1'androsténone nous a été généreusement fourni pär le $\mathrm{D}^{\mathrm{r}} \mathrm{C}_{\mathrm{L}} \mathrm{AUS}$ (Institut de Physiologie, Université Technique de Munich, R.F.A.). L'androsténone tritiée a été obtenue à titre gracieux auprès du Centre de Recherches Synthex (Palo Alto, Californie).

\section{III. - Contrôle olfactif des odeurs sexuelles des viandes}

Le jury de laboratoire effectuant les épreuves olfactives est composé de 6 hommes et 4 femmes. En fonction de la plus ou moins grande disponibilité de chacune de ces personnes, 4 hommes seulement ont participé de façon permanente à l'ensemble des 6 tests olfactifs. L'échelle de notation en 7 points précédemment décrite par BONNEAu et DESMOUlin (I975), comporte la notation du caractère hédonique et du caractère sexuel sensu stricto de l'odeur.

Pour chaque animal étudié, 6 épreuves olfactives sont réalisées :

- Quatre tests sont pratiqués après chauffage à $15^{\circ} \mathrm{C}$ de deux tissus gras (dorsal et périrénal) selon deux méthodes distinctes : chauffage rapide pendant quelques secondes à l'abattoir avec un "fer à souder " selon PATTERson et STINSON (I97I) ou chauffage pendant une heure en tubes fermés selon Dumont et DEsMOULIN (I972).

- Deux autres tests olfactifs sont réalisés sur les viandes au cours de la cuisson au grill d'une série de côtelettes ou en fin de cuisson au four d'un rôti.

\section{IV. - Jugement organoleptique des viandes}

La longe droite de chaque animal est coupée en deux par une section transversale perpendiculaire à la colonne vertébrale et pratiquée entre la $\mathrm{I}_{3}{ }^{\mathrm{e}}$ et $\mathrm{la} \mathrm{I}_{4}^{\mathrm{e}}$ côte. Sur la portion antérieure de la longe on prélève 7 côtelettes dénombrées respectivement $C_{1}, C_{2} \ldots C_{7}$ en allant de l'arrière vers l'avant et sur la portion postérieure 3 rôtis d'environ I $\mathrm{kg}$ dénombrés $R_{1}, R_{2}, R_{3}$ en allant de l'avant vers l'arrière.

\section{IV-I. - Jury de laboratoire}

Après la cuisson d'un rôti $\left(R_{1}\right)$ ou d'une série de 3 côtelettes $\left(C_{1}, C_{2}\right.$ et $\left.C_{3}\right)$, les membres du jury de laboratoire portent un jugement sur l'arôme, la saveur, la tendreté et la jutosité des viandes. Ils donnent en fin une note d'impression générale sur l'échantillon. Les échelles hédoniques utilisées pour chacun des critères comportent 5 niveaux et sont décrites au tableau $\mathrm{I}$.

\section{IV-2. - Jury de consommateurs}

Les autres rôtis et les autres côtelettes sont distribués parmi les membres du personnel du C.N.R.7. Ceux-ci préparent et utilisent les viandes dans les conditions qui leurs sont habituelles. A l'issue de la consommation ils sont invités à 
TABLEAU I

Échelles de notation des caractères organoleptiques par le jury de laboratoire Rating scales for olfactory assessments by laboratory panel

\begin{tabular}{|c|c|c|c|c|c|}
\hline $\begin{array}{l}\text { Note } \\
\text { Score }\end{array}$ & $\begin{array}{l}\text { Arôme } \\
\text { Aroma }\end{array}$ & $\begin{array}{l}\text { Saveur } \\
\text { Flavour }\end{array}$ & $\begin{array}{l}\text { Tendreté } \\
\text { Tenderness }\end{array}$ & $\begin{array}{l}\text { Jutosité } \\
\text { Juiciness }\end{array}$ & $\begin{array}{l}\text { Impression } \\
\text { générale } \\
\text { Overall } \\
\text { opinion }\end{array}$ \\
\hline I & $\begin{array}{l}\text { Très désagréable } \\
\text { Very unpleasant }\end{array}$ & $\begin{array}{l}\text { Très désagréable } \\
\text { Very unpleasant }\end{array}$ & $\begin{array}{l}\text { Très dure } \\
\text { Very tough }\end{array}$ & $\begin{array}{l}\text { Insuffisante } \\
\text { Insufficient }\end{array}$ & $\begin{array}{l}\text { Très défavorable } \\
\text { Very unfavour- } \\
\text { able }\end{array}$ \\
\hline 2 & $\begin{array}{l}\text { Désagréable } \\
\text { Unpleasant }\end{array}$ & $\begin{array}{c}\text { Désagréable } \\
\text { Unpleasant }\end{array}$ & $\begin{array}{l}\text { Dure } \\
\text { Tough }\end{array}$ & $\begin{array}{l}\text { Faible } \\
\text { Slight }\end{array}$ & $\begin{array}{l}\text { Défavorable } \\
\text { Unfavourable }\end{array}$ \\
\hline 3 & $\begin{array}{c}\text { Sans arôme } \\
\text { particulier } \\
\text { No special } \\
\text { aromat }\end{array}$ & $\begin{array}{l}\text { Sans saveur } \\
\text { particulière } \\
\text { No special } \\
\text { flavour }\end{array}$ & $\begin{array}{l}\text { Moyennement } \\
\text { tendre } \\
\text { Moderately } \\
\text { tender }\end{array}$ & $\begin{array}{l}\text { Moyenne } \\
\text { Moderate }\end{array}$ & $\begin{array}{l}\text { Moyenne } \\
\text { Moderate }\end{array}$ \\
\hline 4 & $\begin{array}{l}\text { Moyennement } \\
\text { agréable } \\
\text { Modevately } \\
\text { pleasant }\end{array}$ & $\begin{array}{l}\text { Moyennement } \\
\text { agréable } \\
\text { Moderately } \\
\text { pleasant }\end{array}$ & $\begin{array}{l}\text { Tendre } \\
\text { Tender }\end{array}$ & $\begin{array}{l}\text { Satisfaisante } \\
\text { Satisfactory }\end{array}$ & $\begin{array}{l}\text { Favorable } \\
\text { Favourable }\end{array}$ \\
\hline 5 & $\begin{array}{l}\text { Très agréable } \\
\text { lery pleasant }\end{array}$ & $\begin{array}{l}\text { Très agréable } \\
\text { l'ery pleasant }\end{array}$ & $\begin{array}{l}\text { Très tendre } \\
\text { l'ery tender }\end{array}$ & $\begin{array}{l}\text { Abondante } \\
\text { Abundant }\end{array}$ & $\begin{array}{l}\text { Très favorable } \\
\text { Very favourable }\end{array}$ \\
\hline
\end{tabular}

porter leur jugement (très favorable (3), favorable (2) ou défavorable (I)) sur l'odeur, la saveur, la tendreté et la tenue à la cuisson du morceau. Leur impression générale est également demandée en référence à une échelle à 5 niveaux : très favorable (5), favorable (4), moyenne (3), légèrement défavorable (2), très défavorable (I).

\section{V. - Jugement organoleptique des produits transformés}

Sur une partie des animaux $(N=30)$ la moitié de la carcasse est transformée en produits de charcuterie à 1'École Supérieure des Métiers et de la Viande. 'Trois types de produits sont ainsi préparés : jambon cuit (dit jambon de Paris) saucisson cuit et rillettes.

Le jury de laboratoire réalise un jugement hédonique d'ensemble de ces trois produits en référence à une échelle à 5 niveaux, il est également invité à préciser la nature de tout caractère anormal qu'il serait amené à déceler à l'issue de la consommation des produits.

Par ailleurs deux de ces produits (jambon cuit et rillettes) sont distribués 
aux membres du personnel du C.N.R.Z. (jury de consommateurs) qui doivent les juger à leur domicile dans les mêmes conditions que le jury de laboratoire. En moyenne chaque produit est dégusté par 4 à 5 familles différentes.

\section{VI. - Exploitation statistique des résultats}

Le degré de liaison entre une variable qualitative (notation d'odeur) et une variable quantitative (teneur en androsténone) est établi en calculant le coefficient de corrélation des rangs de Spearman (SIEGEL, I956). La notation des défauts sexuels est considérée selon 3 niveaux (absence d'odeur sexuelle, odeur sexuelle légère ou odeur sexuelle prononcée).

La comparaison entre les notations obtenues au cours des différents tests, ou pour les divers lots d'animaux est établie à l'aide du test 2 I (ARBonNier, I966).

\section{Résultats}

\section{I. - Composition des tissus gras}

\section{I-I. - Teneur en androsténone des graisses dorsales des porcs mâles entiers}

Les valeurs moyennes de la teneur en androsténone des graisses dorsales observées chez les LB (o,6I ppm) et chez les PP (I,75 ppm) sont significativement différentes $(P<0,0 I)$. Les variations intrarace sont cependant très fortes puisque le coefficient de variation est de $56 \mathrm{p}$. Ioo chez les LB et de $68 \mathrm{p}$. Ioo chez les PP. Les teneurs en androsténone élevées (supérieures à I ppm) concernent $73 \mathrm{p}$. Ioo des PP contre I3 p. Ioo seulement des $L_{4} B$.

La teneur en androsténone des graisses est assez indépendante de l'âge et du poids vif chez les LB et peu liée à ces deux critères chez les PP plus âgés (tab1. 2). La relation avec le poids des testicules est peu étroite $(r=+0,44$ à $+0,45)$. Par ailleurs, les corrélations les plus étroites sont établies négativement avec la teneur en muscle chez les LB $(r=-0,52)$ et positivement avec le gain de poids en finition $(r=+0,54)$ chez les PP.

\section{I-2. - Composition en acides gras des graisses}

Les différences de composition en acides gras des graisses en fonction du tissu, du type sexuel et de la race sont rapportées au tableau 3. Les graisses dorsales significativement plus insaturées que les graisses périrénales, présentent une teneur plus élevée en acides palmitoléique (CI6:I) et oléique (CI8: I). Les teneurs en acides myristique ( $\left.\mathrm{CI}_{4}: 0\right)$ et linoléique (CI $8: 2$ ) ne diffèrent pas dans les deux tissus.

Le degré d'insaturation des graisses ne diffère pas selon le type sexuel ou selon 1a race. En effet, la teneur plus élevée en acide linoléique, observée chez les entiers par rapport aux castrés $(\mathrm{P}<0,00 \mathrm{I})$ ou chez les $\mathrm{PP}$ par rapport aux $\mathrm{LB}(\mathrm{P}<0,00 \mathrm{I})$ est compensée par une diminution de la teneur en acide oléique $(P<0,0 I)$. Les différences entre race pout la teneur en CI $8: 2$ sont plus importantes chez les mâles entiers que chez les castrats (Interaction Race $\times$ Type sexuel très hautement significative : $\mathrm{P}<\mathrm{O}, \mathrm{OOI}$ ). 
M. BONNEAU, B. DESMOULIN, B. L. DUMONT

TABLEAU 2

Coefficients de corvélation entre la teneur en androsténone des graisses et divers critères zootechniques et de composition corporelle

Correlation coefficients between androstenone content in fat and zootechnical and body composition parameters

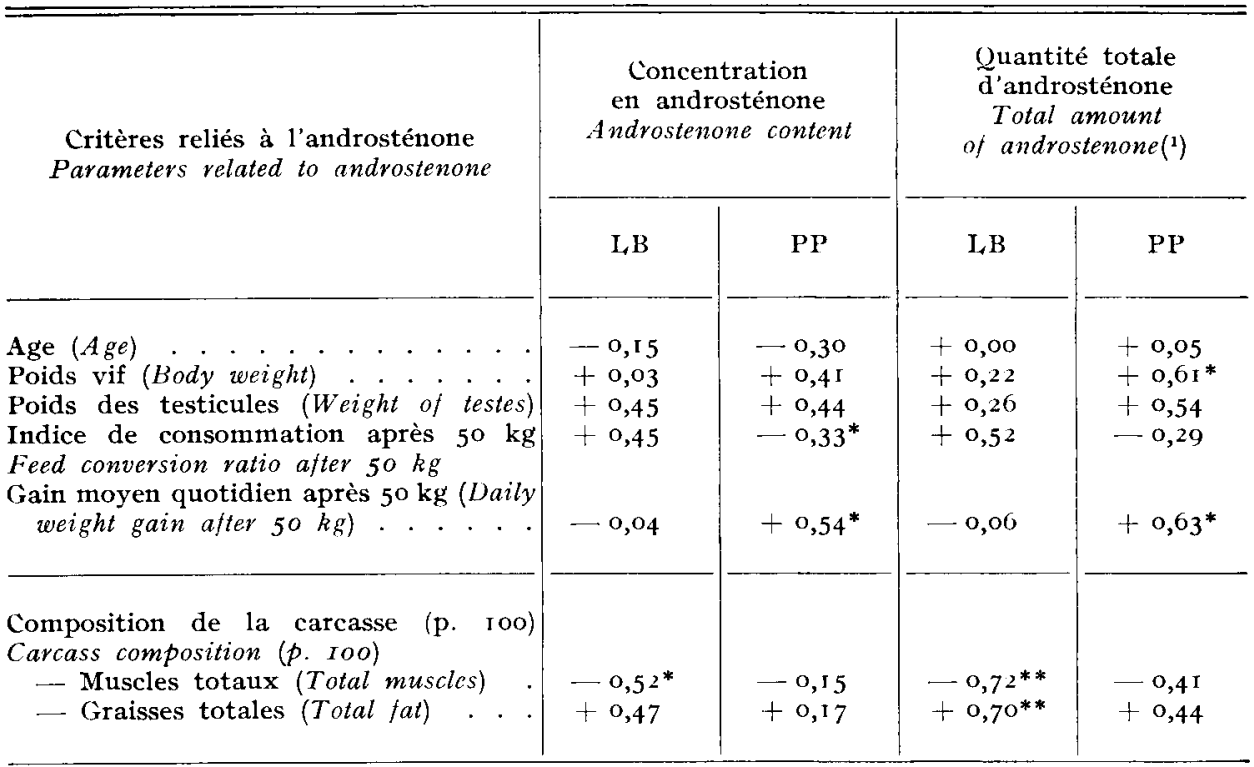

(1) Valeur estimée en multipliant la concentration en androsténone par le poids des graisses dans la carcasse. (Estimated value : Audrostenone content $\times$ weight of fatty tissue in the carcass).

$+\mathbf{P}<0,10 ; * \mathrm{P}<0,05 ;{ }^{* *} \mathrm{P}<0,01$.

La composition en acides gras des graisses est reliée de façon étroite au degré d'adiposité des animaux (tabl. 4). Pour les deux types sexuels la teneur en Cr $8: 2$ est reliée négativement au pourcentage de graisses dans la carcasse. On observe en outre chez les mâles entiers, une relation positive entre ce dernier critère et les teneurs en acides oléique (pour les deux tissus), palmitoléique et stéarique (pour le tissu gras dorsal).

Les corrélations entre la teneur en androsténone et la composition en acides gras des graisses ont été calculées pour 9 porcs. Dans le cas des graisses dorsales, la teneur en stéroïde est reliée positivement au degré d'insaturation des graisses $(r=+0,79 ; \mathrm{P}<\mathrm{o}, \mathrm{OI})$. Cette relation concerne plus particulièrement les acides CI6 : I $(r=+0,5 \mathrm{I})$ et $\operatorname{CI} 8:$ o $(r=-0,80 ; \mathrm{P}<0,05)$.

\section{II. -- Jugement des odeurs sexuelles au cours des différents tests olfactifs}

II-I. - Degré de liaison entre la notation des odeurs sexuelles et la teneur en androsténone des graisses des mâles entiers

Dans le tableau 5 sont rapportés séparément les résultats concernant les juges I et 2 dont les notations sont les mieux reliées à la teneur en androsténone et ceux obtenus en considérant l'ensemble des membres du jury. 


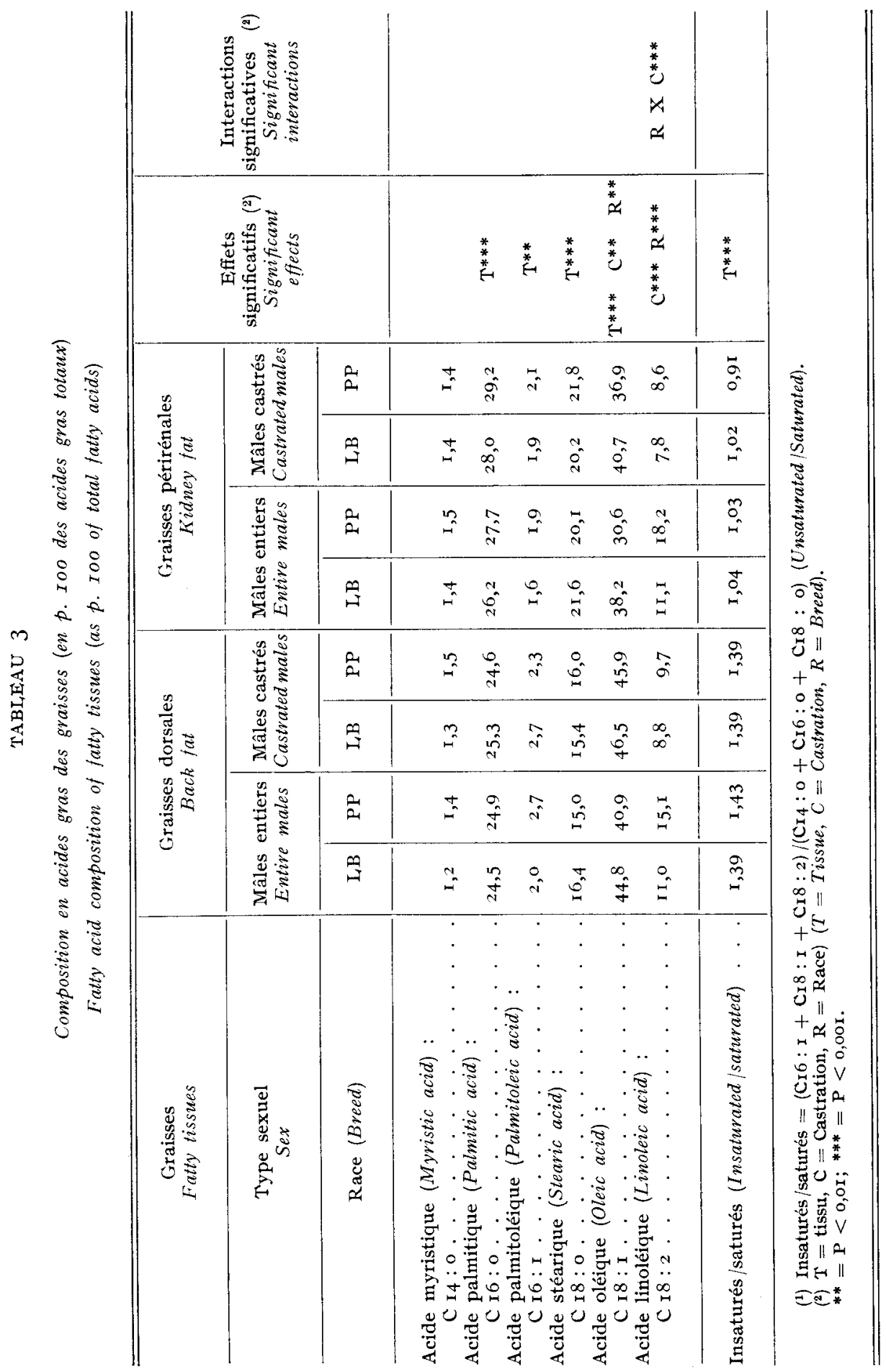




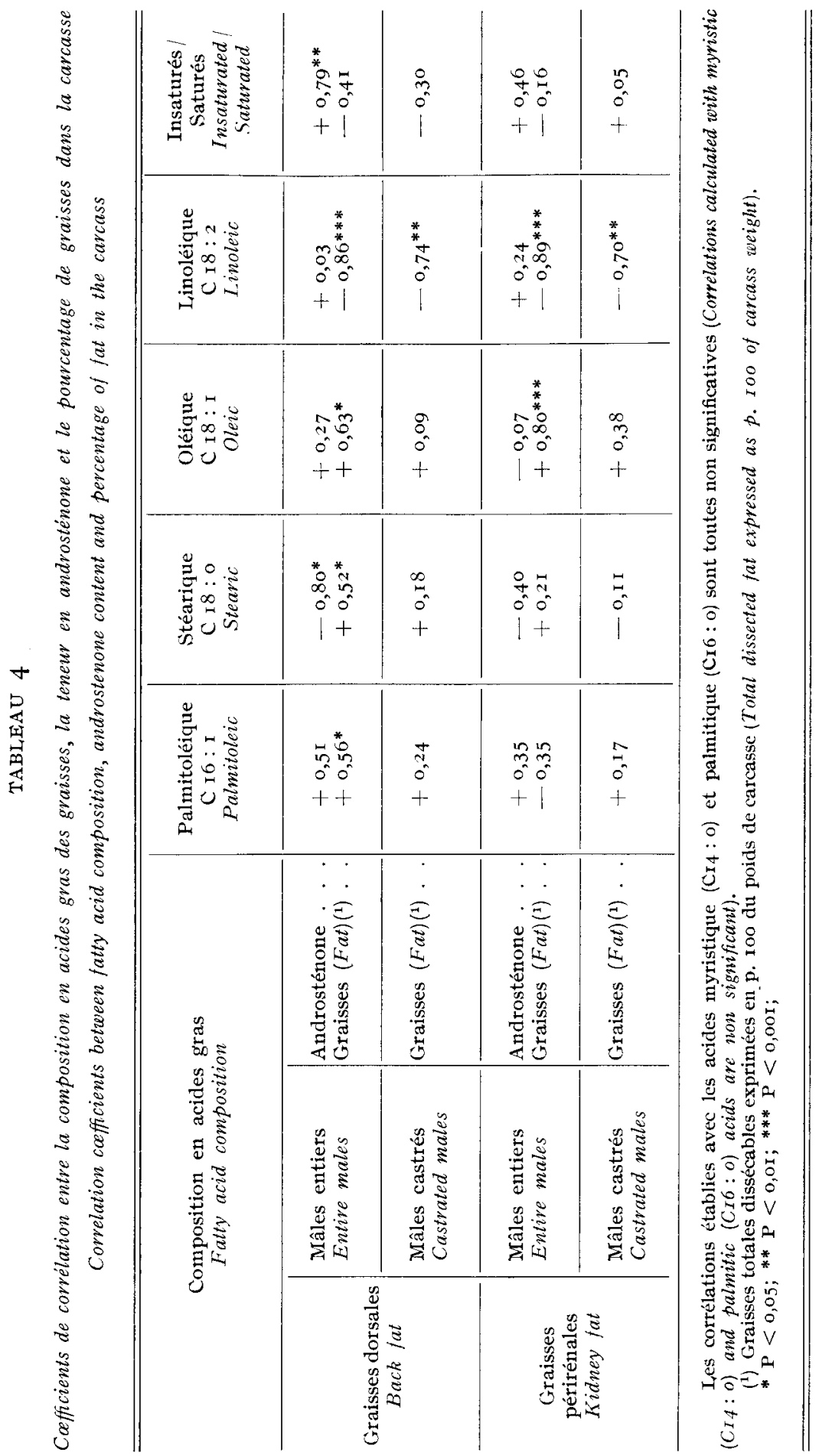


TABLEAU 5

Relations entre la teneur en androsténone des graisses

et la notation de l'intensité des odeurs sexuelles chez les porcs males entiers

Relationships between fat androstenone content and rate of intensity of boar taint in boars

\begin{tabular}{|c|c|c|}
\hline & $\begin{array}{l}\text { Ensemble du jury } \\
\text { Whole panel }\end{array}$ & $\begin{array}{l}\text { Juges I et } 2 \\
\text { Panel members } I \text { and } 2\end{array}$ \\
\hline $\begin{array}{l}\text { Nombre moyen de données par test (Mean num- } \\
\text { ber of data per test) . } . .+.+. \\
\end{array}$ & 137 & 45 \\
\hline $\begin{array}{l}\text { Chauffage des graisses dorsales (Back fat heating) } \\
\text { - au fer à souder (With soldering iron) } \\
\text { - en tubes fermés (in closed tubes)... }\end{array}$ & $\begin{array}{l}+o, 4 I^{*} \\
+o, 15\end{array}$ & $\begin{array}{l}+0,57^{* *} \\
+0,52^{* *}\end{array}$ \\
\hline $\begin{array}{l}\text { Chauffage des graisses périrénales (Kidney fat } \\
\text { heating) : } \\
\text { - au fer à souder (with soldering iron) . . } \\
\text { - en tubes fermés (in closed tubes). . . . }\end{array}$ & $\begin{array}{l}+0,34^{+} \\
+0,31^{+}\end{array}$ & $\begin{array}{l}+0,3^{6+} \\
+0,4^{6 *}\end{array}$ \\
\hline 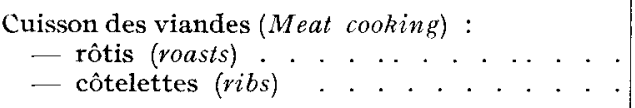 & $\begin{array}{l}+0,44^{*} \\
+0,34^{+}\end{array}$ & $\begin{array}{l}+0,54^{* *} \\
+0,36^{+}\end{array}$ \\
\hline
\end{tabular}

$+\mathrm{P}<0, \mathrm{IO} ; * \mathrm{P}<0,05 ; * * \mathrm{P}<0,01$.

Au cours des tests de chauffage des graisses les notations d'odeurs portées sur les graisses dorsales sont plus dépendantes de la teneur en androsténone que celles portées sur les graisses périrénales. Pour les premières, le chauffage rapide au fer à souder est le test pour lequel les notations sont les mieux reliées à la teneur en androsténone.

Au cours de la cuisson des viandes, les notations des odeurs sexuelles sont liées de façon plus étroite à la teneur en androsténone dans le cas du rôti que pour les côtelettes.

\section{II-2. - Comparaison des notations d'odeurs obtenues au cours des différents tests}

Le choix des tissus et des méthodes de chauffage ou de cuisson détermine des variations importantes dans la notation des odeurs sexuelles. Le calcul des valeurs de $2 \widehat{I}$ permet de comparer les répartitions des avis (dans les 3 classes d'odeur précédemment décrites) obtenues au cours des divers tests. Les valeurs de $2 \widehat{\mathrm{I}}$ faibles, correspondant à des probabilités élevées, indiquent que les répartitions diffèrent peu. A l'inverse les valeurs de $2 \widehat{\mathrm{I}}$ élevées, correspondant à des probabilités faibles, montrent que les répartitions sont significativement différentes.

L,es notations obtenues sur les graisses périrénales par l'une ou l'autre des 2 méthodes de chauffage diffèrent peu $(\mathrm{P}>0,20$ à $\mathrm{P}>0,90)$. Par contre, dans 
le cas des graisses dorsales, 1 'in fluence du mode de chauffage est importante $(\mathrm{P}<$ $0,05$ à $\mathbf{P}<0,00 \mathrm{r})$. De même les notations obtenues au cours de la cuisson des rôtis ou des côtelettes sont significativement différentes $(\mathrm{P}<0,05$ à $\mathrm{P}<0,00 \mathrm{I})$.

Finalement (tab1. 6), les différents tests peuvent être regroupés en 3 catégories homogènes $(\mathrm{P}>0,10$ à $\mathrm{P}>0,90)$ et significativement différentes entre elles

TABIEAU 6

Comparaison des notations de l'intensité des odeurs sexuelles au cours des différents tests olfactifs Comparison of boar taint intensity ratings during different olfactory tests

\begin{tabular}{|c|c|c|c|c|c|c|}
\hline $\begin{array}{c}\text { Juges } \\
\text { Panel members }\end{array}$ & \multicolumn{3}{|c|}{$\begin{array}{c}\text { I et } 2 \\
I \text { and } 2\end{array}$} & \multicolumn{3}{|c|}{$\begin{array}{c}\text { Ensemble du jury } \\
\text { Whole panel }\end{array}$} \\
\hline Catégories de tests (Categories of tests(1)) & DF-R & C & $\begin{array}{l}\text { PF-PT- } \\
\text { DT }\end{array}$ & DF-R & C & $\begin{array}{l}\text { PF-P'- } \\
\text { DT }\end{array}$ \\
\hline Nombre d'avis (Number of opinions). & I IO & 56 & I 67 & 390 & $20 I$ & 573 \\
\hline $\begin{array}{l}\text { Répartition des avis p. Ioo : } \\
\text { Distribution of opinions (p. Ioo) : } \\
\text { - odeur non sexuelle (no boar taint) } \\
\text { - odeur sexuelle légère (slight boar } \\
\text { taint) . . . . . . . . . . . } \\
\text { - odeur sexuelle prononcée (strong } \\
\text { boar taint) . . . . . . . . . . }\end{array}$ & $\begin{array}{l}55 \\
26 \\
19\end{array}$ & $\begin{array}{l}32 \\
39 \\
29\end{array}$ & $\begin{array}{r}78 \\
14 \\
8\end{array}$ & $\begin{array}{l}49 \\
32 \\
19\end{array}$ & $\begin{array}{l}3 \mathbf{I} \\
32 \\
37\end{array}$ & $\begin{array}{l}62 \\
21 \\
17\end{array}$ \\
\hline $\begin{array}{l}\text { Signification des différences : } \\
\text { Significance of differences : } \\
\text { - entre tests intracatégorie (between } \\
\quad \text { tests, within categories) : . . } \\
\text { - entre catégories (betweencategories) }\end{array}$ & $\begin{array}{l}\mathrm{P}>0,90 \\
\longleftarrow\end{array}$ & $<0$ & $\begin{array}{l}\mathrm{P}>0,5^{\circ} \\
\mathrm{OI} \longrightarrow\end{array}$ & $\mid \begin{array}{l}\mathrm{P}>0,10 \\
\leftarrow \mathrm{P}\end{array}$ & $<0$ & $\stackrel{\mathrm{P}}{\mathrm{OI}} \longrightarrow$ \\
\hline
\end{tabular}

( $\left.{ }^{1}\right)$ Chauffage rapide au fer (F) ou prolongé en tubes (T). Tissu gras dorsal (D) ou périrénal (P). Cuisson des viandes du rôti $(R)$ ou des côtelettes (C) (Heating back fat $(D)$ or kidney fat $(P)$ Heating with soldering iron $(F)$ or in closed tubes $(T)$. Cooking roasts $(R)$ or ribs $(C)$.

$(\mathrm{P}<0,00 \mathrm{I})$. Les notations portées lors du chauffage rapide des graisses dorsales au fer (DF) ou lors de la cuisson du rôti (R) (49 à 55 p. Ioo " non sexuelle ", 26 à 32 p. roo " sexuelle légère " et I 9 p. Ioo "sexuelle prononcée ") sont intermédiaires entre les notations plus sévères portées sur les odeurs de cuisson des côtelettes (29 à 37 p. Ioo "sexuelle prononcée ") et les notations plus libérales obtenues au cours des 3 autres tests ( 62 à 78 p. Ioo d'odeur " non sexuelle "). Les notations portées sur les graisses périrénales se caractérisent, chez les mâles entiers comme chez les castrés, par une fréquence importante d'odeurs désagréables non sexuelles (28 à 35 p. I Oo des avis). 
III. -- Fréquence et intensité des odeurs sexuelles chez les porcs de type hypermusclé

La fréquence des avis d'odeurs sexuelles légères ou prononcées pour les deux types sexuels est reportée au tableau 7 en fonction de la race et du stade d'abattage.

- Dans le cas des mâles entiers LB, la fréquence des défauts sexuels prononcés est relativement faible au stade léger mais elle augmente de façon significative au stade lourd.

- Dans le cas des mâles entiers PP la fréquence des défauts sexuels, (légers ou prononcés, est très élevée au stade léger comme au stade lourd. La répartition des avis entre défauts mineurs ou prononcés est cependant différente selon les stades.

La fréquence des avis d'odeurs désagréables " non sexuelles " est comparable chez les mâles entiers et chez les castrés ( 9 à I 5 p. Ioo lors du chauffage des graisses dorsales au fer, 5 à $8 \mathrm{p}$. Ioo au cours de la cuisson des viandes).

IV. - Notations des caractères organoleptiques des viandes au cours de la dégustation par le jury de laboratoire

Les appréciations sensorielles des viandes au cours de la dégustation par le jury de laboratoire sont rapportées au tableau 8 . Pour chacun des critères étudiés, la notation moyenne reflète bien la répartition des avis dans les différentes classes d'agrément ou de désagrément.

Les effets de la race et du type sexuel sont particulièrement importants pour les critères d'arôme, de saveur et d'impression générale. Les viandes des PP sont moins bien notées que celles des LB $(P<0$,Oor); les viandes des porcs mâles entiers sont jugées plus sévèrement que celles des castrats $(\mathrm{P}<0,00 \mathrm{I})$. En outre, les viandes des animaux entiers sont jugées moins tendres que celles des castrés, particulièrement chez les Piétrains.

Les différences entre morceaux concernent uniquement la tendreté et la jutosité des viandes $(\mathrm{P}<\mathrm{O}, \mathrm{OOI})$ jugées moins satisfaisantes dans le cas des côtelettes que dans celui des rôtis.

I,e calcul des coefficients de corrélation entre les différents critères de la dégustation montre que, chez les mâles entiers, l'impression générale est surtout liée à 1 'arôme $(r=+0,65$ à $+0,84)$ et à la saveur $(r=+0,84$ à $+0,89)$. Dans le cas des mâles castrés, chacun des 4 critères considérés est lié de façon significative à l'impression générale; la saveur semble cependant contribuer de façon prépondérante à la formation de la note globale d'impression générale $(r=+0,75$ à $+0,83)$.

\section{V. - Jugement de l'acceptabilité des viandes par le jury de consommateurs}

Le tableau 9 présente les résultats de l'enquête de consommation effectuée auprès du jury de consommateurs.

Pour les deux races on note, quel que soit le morceau, une influence favorable de la castration sur la qualité générale de la viande : les porcs entiers reçoivent une note moindre pour l'impression générale. On peut penser que cette notation est conditionnées par la moins bonne saveur et surtout par un arôme moins satisfaisant. 
M. BONNEAU, B. DESMOULIN, B. L. DUMONT

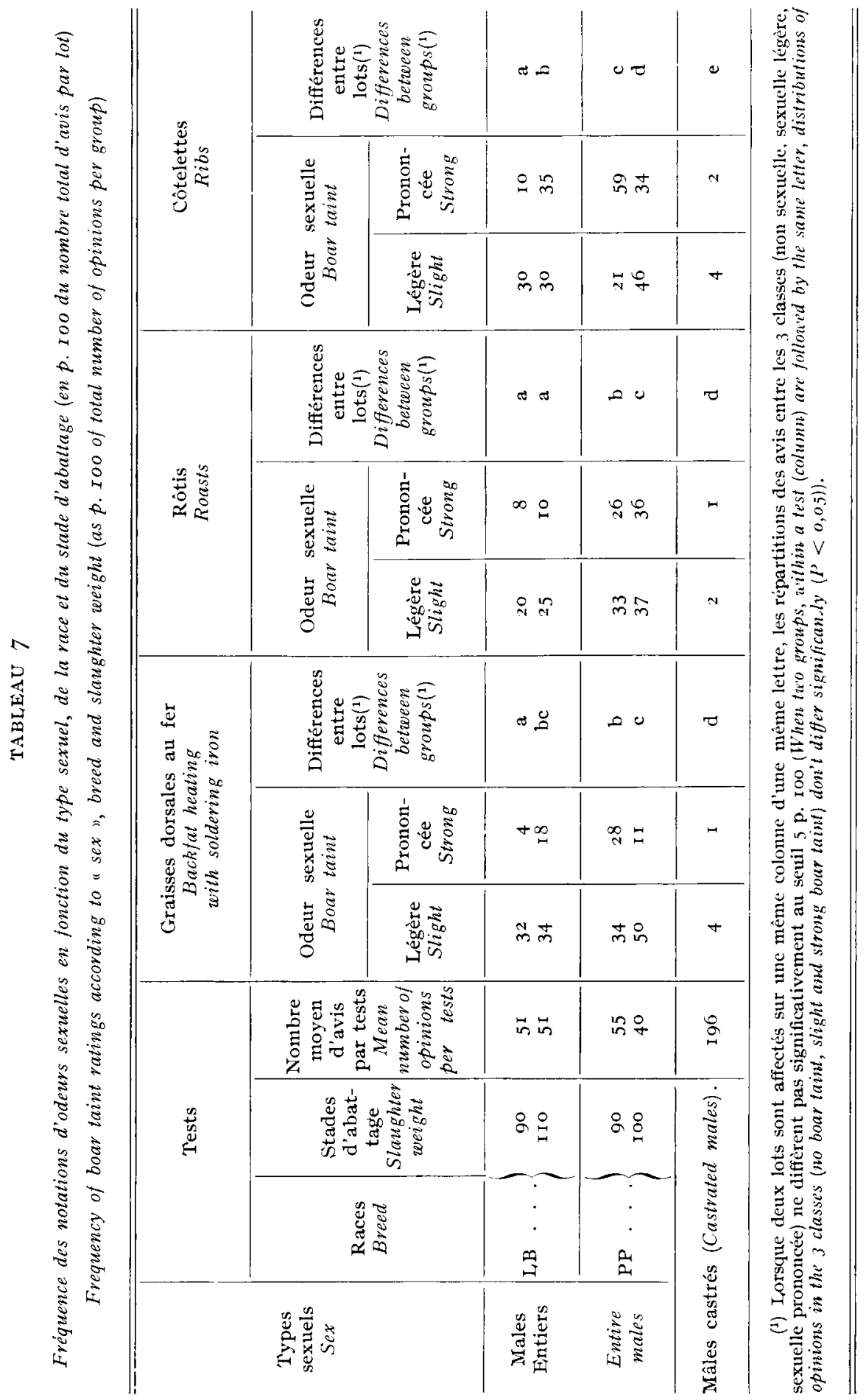




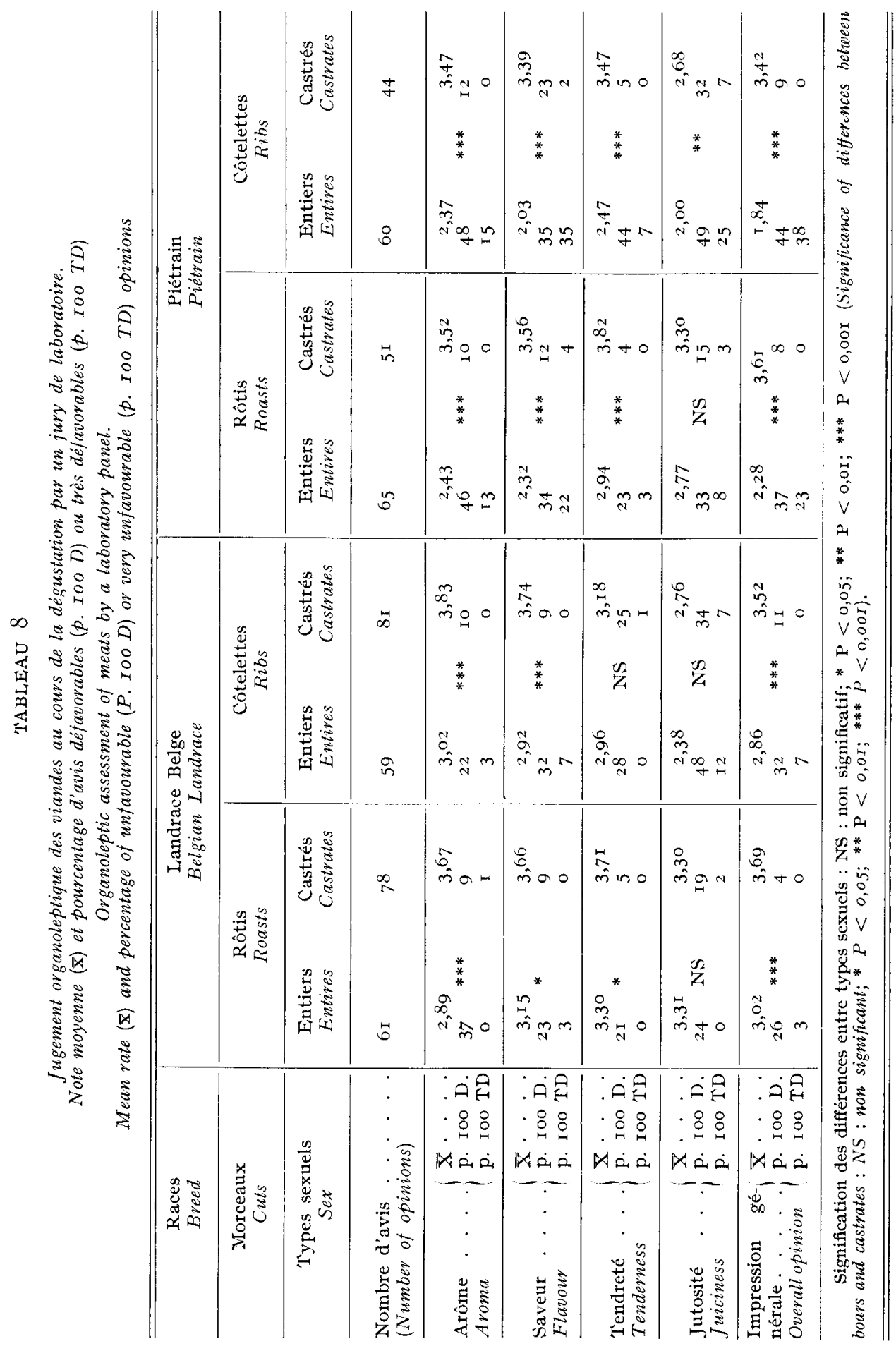




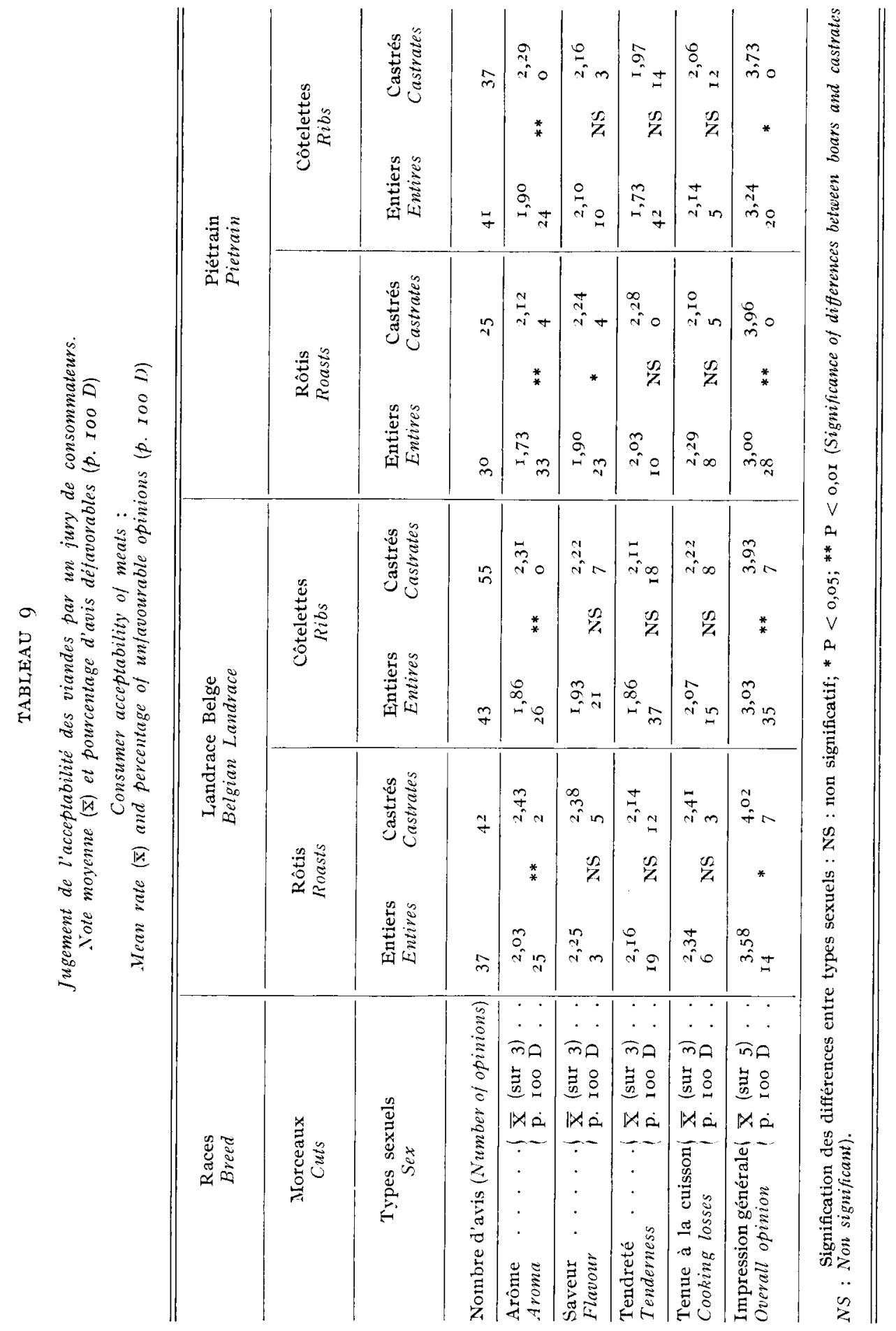


Cette différence entre les mâles castrés et les mâles entiers se traduit par un pourcentage d'insatisfaction important manifesté par le jury des consommateurs à l'encontre des viandes de porcs mâles entiers. Les défauts d'odeurs justifient cette situation pour les deux types de morceaux. La moindre tendreté dans le cas des côtelettes correspond au maximum d'insatisfaction en registrée. Dans le cas des L,B c'est au niveau des côtelettes que la différence entre castrés et entiers est la plus nette pour l'impression générale alors que dans le cas des PP c'est au niveau des rôtis que la différence est la plus marquée.

Entre races les seules différences significatives intéressent, dans le cas des rôtis, la saveur $(\mathrm{P}<0,0 \mathrm{I})$ et l'impression générale $(\mathrm{P}<0,05)$ des $\mathrm{L}, \mathrm{B}$ qui sont supérieurs aux $P P$ pour ces 2 caractères.

\section{VI. - Jugement organoleptique des produits transformés}

Le tableau Io rapporte les résultats concernant l'impression générale du jury de laboratoire pour les différents produits et les divers types d'animaux. Pour le jambon cuit aucune différence n'est mise en évidence selon le type sexuel ou selon la race. Dans le cas des deux autres produits (rillettes et saucisson cuit), on observe une influence de la race chez les mâles entiers (en faveur des LBB) et un effet du type sexuel chez les PP (en faveur des castrats).

TABIEAU IO

Note d'impression générale des produits transformés dégustés par le jury de laboratoive Overall opinion rating of processed meats by the laboratory panel

\begin{tabular}{|c|c|c|c|c|c|c|}
\hline \multirow[t]{2}{*}{$\begin{array}{c}\text { Produits } \\
\text { Processed meats }\end{array}$} & \multicolumn{2}{|c|}{$\begin{array}{l}\text { Jambon cuit } \\
\text { Cooked ham }\end{array}$} & \multicolumn{2}{|c|}{$\begin{array}{l}\text { Rillettes } \\
\text { Rillettes }\end{array}$} & \multicolumn{2}{|c|}{$\begin{array}{l}\text { Saucisson } \\
\text { cuit à l'ail } \\
\text { Cold sausage } \\
\text { with gavlic }\end{array}$} \\
\hline & $\begin{array}{l}\text { Entiers } \\
\text { Entires }\end{array}$ & $\begin{array}{l}\text { Castrés } \\
\text { Castrates }\end{array}$ & $\begin{array}{l}\text { Entiers } \\
\text { Entives }\end{array}$ & $\begin{array}{l}\text { Castrés } \\
\text { Castrates }\end{array}$ & $\begin{array}{l}\text { Entiers } \\
\text { Entives }\end{array}$ & $\begin{array}{l}\text { Castrés } \\
\text { Castrates }\end{array}$ \\
\hline \begin{tabular}{l|l} 
Landrace Belge . $\mathrm{X}$ &. \\
Belgian Landrace & $\mathrm{E} . \mathrm{T}$.
\end{tabular} & $\begin{array}{l}3,42 \\
0,24\end{array}$ & $\begin{array}{l}3,30 \\
0,45\end{array}$ & $\begin{array}{l}3,43 \\
0,36 \mathrm{NS}\end{array}$ & $\begin{array}{l}3,45 \\
0,56\end{array}$ & $\begin{array}{l}3,26 \\
0,78 \mathrm{NS}\end{array}$ & $\begin{array}{l}3,40 \\
0,25\end{array}$ \\
\hline 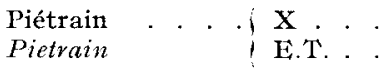 & $\begin{array}{l}3,4 \mathrm{I} \\
0,5 \mathrm{I}\end{array}$ & $\begin{array}{l}3,26 \\
0,33\end{array}$ & $\begin{array}{l}2,74 \\
0,60^{*}\end{array}$ & $\begin{array}{l}3,45 \\
0,32\end{array}$ & $\begin{array}{l}2,80 \\
0,71 *\end{array}$ & $\begin{array}{l}3,92 \\
0,6 \mathrm{I}\end{array}$ \\
\hline $\begin{array}{l}\text { Signification des différences } \\
\text { entre races (Significance of } \\
\text { differences between breeds) }\end{array}$ & NS & NS & * & NS & * & NS \\
\hline
\end{tabular}

$\mathrm{X}$ : Note moyenne (Mcan rate).

E.T. : Écart-type (Standard deviation). 
Au niveau du jury de consommateurs, l'impression générale est notée en moyenne 4,36 (entiers) et 4, Io (castrats) dans le cas du saucisson cuit : pour les rillettes les notes sont respectivement 3,72 pour les entiers et 4, Io pour les castrats. On observe ainsi, comme pour le jury de laboratoire, une tendance à ce que les consommateurs préfèrent les jambons des porcs entiers à ceux des castrats alors qu'il jugent les rillettes de castrats supérieures à celles des entiers. Les différences entre races et entre types sexuels ne sont cependant pas significatives.

En fonction du produit considéré, des caractères anormaux de flaveur ont été précisés pour 0,7 à 4,2 p. Ioo des avis dans le cas du jury de laboratoire et pour o à r 3.9 p. roo des avis dans le cas du jury de consommateurs.

\section{Discussion}

\section{I. - Composition des graisses}

L'échantillon des porcs mâles entiers étudiés au cours de cet essai est très hétérogène en ce qui concerne les teneurs en androsténone des graisses. La fréquence des concentrations en stéroïde supérieures à I ppm chez les LB (13 p. Ioo des porcs) est comparable à celle rapportée par CLAUS (1977) chez les animaux Landrace Allemand (22 p. IOO) et légèrement inférieure à celle indiquée par MALMFors et LuNdSTROM (I977, communication personnelle) chez les Landrace Suédois (3I p. I0o) Par contre, les mâles entiers PP constituent une population très originale présentant des concentrations en stéroïde très élevées (73 p. Ioo des animaux au-dessus de I $\mathrm{ppm}$ ). Ce résultat pourrait être relié à la précocité sexuelle plus grande des PP par rapport au LB ou aux Landrace Français, chez les mâles comme chez les femelles (ÉTIENNE et LEGAUI,T, I974).

Le degré d'insaturation des graisses ne diffère pas significativement entre les deux races étudiées. On observe cependant une augmentation de la teneur en acide linoléique et une diminution de celle en acide oléique chez les PP. WOOD (I 973) obtient des teneurs plus élevées en acides linoléique et oléique chez les PP, comparativenient aux Large White.

Le degré d'insaturation des graisses des porcs entiers ne diffère pas significativement de celui des graisses de castrats. En effet, en accord avec les résultats de Maimfors, Lunidstrom et Hansson (I978), l'augmentation de la teneur en acide linoléique est compensée par la diminution de la concentration en acide oléique.

Les variations de la composition en acides gras des graisses peuvent résulter des différences de développement du tissu adipeux. En effet, comme 1'avaient déjà constaté PASCAL et al. (I975), la teneur en acide linoléique, non synthétisé par l'animal, est d'autant plus élevée que le pourcentage de graisses dans la carcasse est plus faible $(r=-0,70$ à - o,89). Ce résultat peut expliquer les différences de composition des graisses selon le type sexuel et selon la race.

\section{II. - Relation entre la composition des graisses et l'importance des défauts sexuels}

Une liaison significative est mise en évidence entre la composition en acides gras et la teneur en androsténone des graisses. L'observation selon laquelle les graisses de porcs mâles entiers présentant des odeurs sexuelles sont plus insaturées et, en particulier, plus riches en acide linoléique que celles des porcs mâles indemnes de défauts (Bonneau et Desmoulin, I975) est confirmée par NEIDINIUC, Anto- 
NARU et Contora (I978), ainsi que par Jonsson et Wismer Pedersen (I974) qui ont calculé une corrélation $r=+0,65$ entre l'intensité des odeurs sexuelles et l'indice d'iode des graisses. Par contre, selon Malmfors et Andresen (1975), les liaisons entre la teneur en androsténone et la composition en acides gras des graisses sont faibles et non significatives. AlLEN, BRAY et CASSENS (I967), KOCH, Parr et Merkel, (I968), Jonsson et Wismer Pedersen (r974), Nedeiniuc, ANTONARU et CONTORA (I978) montrent en outre que les graisses des porcs mâles entiers sont plus insaturées que celles des castrats. La relation entre la teneur en androstérone et le degré d'insaturation des graisses peut résulter de l'impact des androgènes sur le métabolisme des graisses. BuTrov (1973) a montré en effet que l'administration d'androgènes à des castrats entraîne une augmentation du degré d'insaturation des graisses.

La fréquence et l'intensité des odeurs sexuelles diffèrent sensiblement selon les tissus et selon les méthodes de chauffage ou de cuisson utilisées :

- Lors du chauffage des graisses périrénales, la fréquence d'odeurs désagréables non sexuelles constitue une gêne importante pour l'appréciation des défauts sexuels.

- I Dans le cas des graisses dorsales, en accord avec MaArse, MoERman et WAISTRA (I972), le test de chauffage prolongé en tubes est moins révélateur des défauts sexuels que celui pratiqué à l'aide d'un " fer à souder ". Les différences entre ces deux méthodes pourraient s'expliquer par la formation de composés de natures différentes.

- Lors de la cuisson des viandes, la répartition des avis dans le cas du rôti est très comparable à celle obtenue lors du chauffage des graisses dorsales au fer. Par contre, le jugement des odeurs de cuisson des côtelettes est beaucoup plus sévère que ne le prévoit le test au fer à souder. Ainsi, dans ces conditions, le seul test de chauffage rapide des graisses dorsales au fer à souder ne constitue pas un prédicteur sécurisant des défauts sexuels révélés lors de la cuisson des viandes.

Les corrélations établies entre l'intensité des odeurs sexuelles révélées lors du chauffage des graisses dorsales au fer à souder et la teneur en androsténone des graisses $(r=+0,4 \mathrm{I} \grave{\mathrm{a}}+0.57)$ sont comparables à celles obtenues par MALMFOrs et ANDRESEN (I975, $r=+\mathbf{0 . 5 I}$ ) ou par NEWELL et al. (I973; $r=+0,53$ ). Ces liaisons peu étroites indiquent que l'androsténone n'est pas le seul responsable des défauts d'odeurs sexuelles. Les corrélations obtenues par PATTERson et STINSON (I97I) sur des échantillons de graisses auxquels des quantités connues d'androsténone ont été ajoutées sont en effet meilleures $(r=+0,76)$. Thompson et Pearsor (I977) montrent de même que l'androsténone n'est pas le seul composé contribuant aux odeurs sexuelles. Ces mêmes auteurs (Thompson, Pearson et Banks, 1972) ont mis en évidence la présence d'autres composés stéroïdes (à odeur de musc ou à odeur d'urine) de la famille des CI9 $\Delta \mathrm{I} 6$, dans les graisses d'un verrat adulte. Par ailleurs, selon MAArse et al. (I972), le stockage préférentiel de scatol (à odeur fécale) dans les graisses des porcs mâles entiers pourrait jouer un rôle notable dans la notation des odeurs sexuelles.

\section{III. - I'ariations de l'importance des défauts sexuels en fonction du type génétique}

L'influence de la race sur la fréquence et l'intensité des défauts sexuels est importante puisque les mâles entiers PP sont jugés plus défectueux que leurs homologues LB. Des différences entre races sont également mentionnées par 
Rhodes (I97I) et par Malmfors et Hansson (I974), selon lesquels les défauts sexuels sont plus importants chez des animaux de type Landrace que chez les porcs de type Large White ou Yorkshire. La possibilité de contrôler les facteurs génétiques est démontrée par Aısing et al. (I977) : la sélection des porcs à partir de la concentration en stéroïde des tissus gras prélevés par biopsies permet après une seule génération, de différencier deux lignées. Mais les performances de croissance de la lignée à faible teneur en stéroïde ayant été nettement réduites, l'intensité de sélection à exercer contre le caractère sexuel indésirable reste à préciser.

L'opportunité de la castration des porcs mâles selon les types génétiques et la fréquence des défauts sexuels des viandes mâles doit donc être envisagée en tenant compte des différences d'aptitudes à l'emploi des viandes. Pour la consonimation en porc frais, les limites d'utilisation du porc mâle entier de race PP dès le stade de $90 \mathrm{~kg}$ sont beaucoup plus importantes que celles du porc I,B.

IV. - Comparaison des jugements obtenus auprès du jury de laboratoire et auprès du jury de consommateurs

D'une manière générale quels que soient les produits (viandes fraîches, rôtis, côtelettes et produits fabriqués) le jury des consommateurs apparaît comme beaucoup moins sévère que le jury de laboratoire. Ce dernier présente l'avantage d'être beaucoup plus entraîné et par là plus discriminant. Au plan de l'acceptabilité des viandes par le grand public on peut donc estimer qu'on dispose d'un coefficient de sécurité considérable en prenant comme référence les opinions du jury de laboratoire.

Quoiqu'il en soit on doit apprécier les conséquences commerciales pratiques, au plan de l'acceptabilité des viandes, des taux d'insatisfaction révélés dans cette étude : on pourrait admettre qu'un pourcentage de jugements défavorables de 5 à Io $\mathrm{p}$. Ioo à l'encontre d'un produit est déjà de nature à compromettre le marché de ce produit. Au-delà, le taux d'insatisfaction serait tel que la distribution hésiterait sans doute à envisager une commercialisation régulière. Si 1'on retient ce critère comme base de comparaison des produits, on voit qu'en règle générale pour les viandes fraîches, les animaux entiers des races considérées posent un réel problème. Par contre, dans le cas des produits transformés considérés dans cet essai, qui sont consommés froid, les différences d'acceptabilité entre les viandes des animaux entiers et celles des castrats sont très atténuées. Des travaux complémentaires doivent être effectués sur l'aptitude à la transformation des viandes de porcs mâles entiers.

Accepté pour publication en octobre 1978

\section{Remerciement}

Nous tenons à remercier le $\mathrm{D}^{\mathrm{r}} \mathrm{C}_{\mathrm{L} A \mathrm{US}}$ (Institut de Physiologie, Université Technique de Munich, R.F.A.) qui a accueilli l'un d'entre nous dans son laboratoire pour l'initier à la méthode de dosage radioimmunologique de l'androsténone et qui lui a permis d'effectuer les déterminations de teneur en stéroïde du présent essai.

Nous sommes également reconnaissants à M. Raracco de l'École Supérieure des Métiers de la Viande (Paris) d'avoir assuré la réalisation des différents types de produits transformés. 


\section{Summary}

\section{Organoleptic quality of meat from entire or castrated male pigs: composition of fatty tissues and boar taint in "double muscled" breeds}

Seventy two male pigs (entires or castrates) of Belgian Landrace (BL) or Piétrain (PP) breed were slaughtered at either go $\mathrm{kg}$ or I oo- I o $\mathrm{kg}$ live weight.

Very important interindividual differences were encountered within breed for androstenone content in back fat. However, average steroid concentration was higher for PP (I.75 ppm) than for BL boars (0.6I ppm). Androstenone content in back fat was highly related to insaturated saturated fatty acid ratio $(r=+0.79 ; \mathrm{P}<0.0 \mathrm{I})$. Moreover, linoleic acid concentration of fatty tissues was higher for entires than for castrates and for PP than for BL (table 3). I,inoleic acid content was negatively related to percentage of fatty tissue in carcass $(r=--0.86$ to - 0.89 ; $\mathrm{P}<0.00 \mathrm{I})$.

Olfactory assessments of fatty tissues and meats were realised by a laboratory panel. Relationship between androstenone content and boar taint intensity depended on the tests and on the panel members (table 5).

The highest correlations were obtained for olfactory assessment of backfat during heating with a soldering iron $\left(r_{8}=+0.4\right.$ I to +0.57$)$ and of roasts during cooking $\left(\gamma_{s}=+0.44\right.$ to +0.54$)$ Frequency of boar taint was very high as early as $90 \mathrm{~kg}$ live weight in PP boars (table 7); in BI, boars it was slight at $90 \mathrm{~kg}$ but clearly increased at I o $\mathrm{kg}$.

Organoleptic assessments of fresh (roasts and ribs) or processed meat (cooked ham, riliettes and cold sausage with garlic) were realised by the laboratory panel and by a consumer panel. Concerning fresh meat, opinions from the two panels were less favourable for entires than for castrates (tables 8 and 9). None of the two panels found differences between boars and castrates for cooked ham. On the other hand, in the case of rillettes and cold sausage with garlic, boar meat was scored less favourably than castrate's by the laboratory panel.

\section{Références bibliographiques}

AlitiN E., Bray R. W., Cassens R. G., 1967. Changes in fatty acid composition of porcine muscle lipid associated with sex and weight. $J$. Fd. Sci., 32, 26-29.

Alsing W, Claus R., Pirchner F., Willeke H., i977. Selektions experiment auf Ebergeruch.

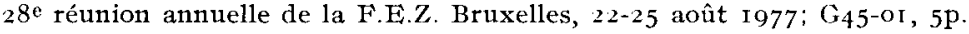

ANDRESEN O, BAKKE H., I975. $5 \alpha$-Androsténone in fat from boars selected for rate of gain and thickness of backfat and from boars used in artificial insemination service. Acta vot. scand., 16, 492-502.

Arbonnier P., 1966. L'analyse de l'information : aperçu théorique et application à la loi multimoniale. Ann. Sci. For., 23, 950-1020.

Bonneau M., Desmoulin B., r975. Comparaison des méthodes d'estimation des défauts d'odeur sexuelle des viandes de pores mâles entiers ou castrés de type Landrace Belge ou Piétrain. Journées Rech. Porcine en France, 7, 2 I 5-223, I.N.R.A.-I.T.P. éd., Paris.

Butrov E. V., 1973. Effect of steroid hormone on the fatty acid composition of hog muscle lipids. Bull. Vses. Nautch. Issl. Inst. Fiziol. I Biokh. Sel'sk, 7 (4), 54-56.

CASTEEIS M., EECKHOUT W., BEKAERT H., BUYSSE F., r974. L'aptitude des verrats à la production de viande : 2 odeur et goût de la viande. Revue A gric. Brux. 1, I 70- I 89.

C'LAUS R., 1974. Dosage radioimmunologique du $5 \alpha$-Androst-I 6-ene-3-one, stéroïde responsable de l'odeur de verrat, dans le tissu adipeux des porcs. C.R. Acad. Sci. Paris 278, 299-302.

CLAUS R., 1977. Pheromone bei Säugetieren unter besonderer Berücksichtigung des Fbergeruchstoffes und seiner Beziehung zu anderen Hodensteroiden. Thèse de l'Université Technique de Munich, $33^{\circ} \mathrm{p}$.

Desmoulin B., Dumont B. L., JAcquet B., I97 I. Le porc mâle de race Large White : aptitudes à la production de viande. Journées Rech. Porcine en France, 3, I87-196, I.N.R.A.-I.T.P. éd., Paris. 
Dfamollin B., Dumont B. L., Pascal G., i973. Qualités des carcasses et des viandes de porcs nuâles entiers de 120 et $140 \mathrm{~kg}$ : addition de BHT aux régimes maïs-soja. Journées Rech. Porcine en France, 5, 20I-209, I.N.R.A.-I.T.P. éd., Paris.

DFsmolin B., Bonneac M., I979. Production des viandes de porcs mâles entiers ou castrés : efficacité alimentaire et composition corporelle chez les races hypermusclées. Ann. Zootech. $28,35-5 \mathrm{I}$.

Demont B. L., Desmoulin B., I972. Contribution à l'étude de la qualité des odeurs de la "viande "de porc. Journées Rech. Porcine en France, 4, 23I-236, I.N.R.A.-I.T.P. éd., Paris.

I'TIENAE M., I,EGaulT C., I974. Influence de la race et du régime alimentaite sur la précocité sexuelle de la truie. Journées Rech. Porcine en France, 6, 57-62, I.N.R.A.-I.T.P. éd., Paris.

HORST P., BADER J., I 969 . Importance of fattening young boars : I-Comparison of fattening and carcass characteristics of boars and sows fattened to I I $\mathrm{kg}$. 2 -Elimination of sexual odour, Zuchtungskunde, 41, 227-26I.

Jonsson P., Wismek-Pendirsen, 1974. Genetics of sex odour in boars. Livest. Prod. Sci., 1, $53-66$.

KOCH D. E., PARR A. F., MERKEL R. A., I968. Fatty acid composition of the inner and Outer layers of porcine Backfat as affected by energy level, Sex and Sire. J. Food Sci., 33, I76-I80.

LFRCir: Z., 1936. Geschlechtsgeruch bei Eberkastraten. Z. Fleisch. Milchhygiene, 46, 4I 7-420.

MaAksi: H., MoERman P. C., Watstra P., I972. Gaschromatografish en organoleptisch onderzoek van vlees en spek van mannelijke varkens en van daarmee bereide rookworst. I.V.O. Rapport $C$. I 80 et rapport $n^{\circ} 3$ Research groep vlees en vleeswaren T.N.O.

Malmfors B., Hansson I., 1974. Incidence of boar taint in swedish Landrace and Yorkshire boars. Livest. Prod. Sci, 1, 41 I-420.

MALMFORS B., ANDRESEN $\varnothing$, I975. Relationship between boar taint intensity and concentration of $5 x$-Androst-r6ène-3-one in boar pleripheral plasma and back fat. Act. A gric. Scand., 25, $92-96$.

Malmfors B., Lendstrom K., Hansson I., 1978. Fatty acid composition of porcine back fat and muscle lipids as affected by sex, weight and anatomical location. Swed. J. Agric. Res., 8, $25-38$.

Nebfiniuc V., Liliana Antosare, Contora N., i 978 . Détermination et correction de l'odeur spécifique de la viande de quelques catégories de porcs. Un nouveau critère de sélection dans l'élevage des porcins. Revta Creterea anim. 28 (5), 17-25.

Neweir. J. A., TUCKFr L. H., Stinson (x. C., Bowlaxin J. P., 1973. Influence of late castration and diethylstilbestrol implantation on performance of boars and on incidence of boar taint. Can. J. anim. Sci., 53, 205-210.

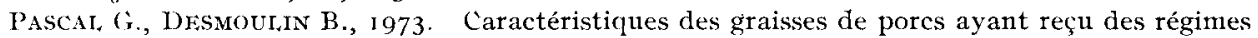
additionnés de BHT. $19^{\circ}$ Réunion des chercheurs en viandes. Paris, F2, 929-946.

Pascai, G., Macaike J. P., Desmollin B., Bonseat M., I975. Composition des graisses des porcs femelles : influence du type génétique (I,F, LB ou PP) et évolution au cours de la croissance (entre $4^{\mathrm{o}}$ et $100 \mathrm{~kg}$ ). Journées Rech. Porcine en France, 7, 203-2J 4, I.N.R.A.-I.T.P. éd. Paris.

PATTERSON R. L. S., I968. $5 \alpha$-Androst-16-ene-3-one : compound responsible for taint in boar fat. J. Si. Fd. Agric, 19, 31-38.

PATTHRSON R.L. S., STINSON G. C., I97I. Rapid estimation of boar taint in pork carcasses. I $7^{\mathrm{t}^{2}}$ réumion Européenne des chercheurs en viandes, Bristol, BI2, I 48 - 53.

RHODEs D. N., I971. Consumer testing of bacon from boar and gilt pigs. J. Sci. Food Agric., 22, $485-490$.

SIFGEI. S., J956. Non parametric statistics for the behavioral Sciences, International student ed., New-York.

Thompsox R. H., Pearson A. M., i 977. Quantitative determination of $5 \alpha$ androst-I6-ene-3-one by gas-chromatography-Mass-spectrometry and its relationship to sex odor intensity in Pork. J. Agric. Fd. Chem., 25, (6), I $24 \mathrm{I}-\mathrm{I} 245$.

Thompsox R. H., Pfakson A. M., Banks K. A., 1972. Identification of some CI9 $\Delta$ I6. Steroids Contributing to sex Odor in Pork. J. Agric. Fd. Chem., 20, (2), I $85^{-1} 89$.

Woon J. D., i 973. The fatty acid composition of back fat from Piétrain and Large White pigs. Anim. Prod., 17, $28 \mathrm{I}-285$. 